\title{
Location Technique based on Pattern Recognition of Radio Signal Strength for Parking Management
}

\author{
Romeo Mark A. Mateo, ${ }^{1}$ Bobby D. Gerardo, ${ }^{2}$ and Jaewan Lee ${ }^{1}$ \\ ${ }^{1}$ School of Electronics and Information Engineering, Kunsan National University, 68 Miryong-dong, Kunsan, Chonbuk 573-701, South Korea \\ ${ }^{2}$ Institute of Information and Communications Technology, West Visayas State University, Luna St., Lapaz, Iloilo City 5000, Philippines \\ Address correspondence to Romeo MarkA. Mateo, rmmateo@kunsan.ac.kr
}

Received 10 June 2011; Revised 14 January 2012; Accepted 23 January 2012

\begin{abstract}
RFID technology is used by various applications to implement smart processing of data. In the parking system, RFID together with wireless sensors are used for identifying cars entering a parking area and monitoring of parking slots. However, there is no means to identify the car parked in a specific slot. This paper proposes a location technique in WSN-based parking management system using radio signal patterns from WSN for a cost-effective method in identifying the car parked in a slot. The proposed pattern recognizer agent (PRA) uses a multilayer perceptron (MLP) to classify the patterns from radio signal strength (RSS) of wireless sensors by associating the signals in a specific parking slot. The implementations provide a training model for RSS patterns and uses classification method in the locating technique. The experiment showed a comparison of the proposed method to other classification algorithms and found that MLP is more accurate classifier and time-efficient in building the classification model.
\end{abstract}

Keywords Please provide the keywords

\section{Introduction}

Most research studies in parking management systems use wireless sensor devices in sensing the presence of cars $[4,5$, 13]. These ubiquitous devices are utilized in monitoring of cars and providing smart processing of information in wireless environment. Relevant services in the parking management system also use the information from wireless sensors for quick notifications of parking events. In the Intelligent Parking System (IPS), real-time information are provided to the drivers who are seeking for parking area to reduce congestion in parking areas, insufficient utilization of the available parking spaces, road congestion caused by spacesearching traffic and safety hazards caused by illegal parking and environmental strains [17]. However, issues of integrating radio frequency identification (RFID) with the wireless sensor networks (WSN) are not studied well. An example of integration issue is how to associate the car presence detector sensors to the car's identification using RFID tags, in which, the system can detect the presence of cars after it parked but cannot tell exactly the identification of car that parked on the slot [5]. This can be solved by location techniques using active signals [18] with the WSN's interactions.

This paper presents the design of our ubiquitous parking management system (UPMS) which is consisted of car active RFID (CARFID) devices, wireless sensor modules and agent components that are interacting to implement the automation of parking management. To solve the problem of identifying a car that parked in a specific slot, the use of pattern recognition of radio signals is proposed. A CARFID sends radio signals to wireless sensor nodes in the parking area and then the pattern recognizer agent (PRA) classifies the signals and associates it to the slot location of the car. The PRA is trained with the received signal strength (RSS) patterns from CARFID in each slot using the MLP structure. The proposed method is implemented in a parking system simulator. The experiment result shows that using multilayer perceptron (MLP) in the proposed method acquires accurate location technique by correctly classifying the current radio signal patterns to the parking slot.

The rest of this paper is organized as follows: Section 2 discusses the related works in parking management system and statistical approaches used for RSS-based location technique. Section 3 presents our parking management system using the ubiquitous devices in wireless environment and agent system for dissemination of information in the wired network environment. Section 4 shows the components for the location technique and the integration of MLP to the PRA for radio signal recognition. In Section 5, the implementation and performance evaluation of our work are shown and, finally, Section 6 concludes our contribution.

\section{Related work}

\subsection{Parking management systems}

In parking management, policies are made to ensure the use of available parking spaces. The socioeconomic implications are considered in deciding appropriate 
location and maintaining parking areas [3] to prevent under priced parking and overcrowding facility. Multiple linear regression (MLR) and geographically weighted regression (GWR) models are used in [8] to estimate parking demands in areas with paid short stay parking systems. Wireless sensors are used in the parking management systems to provide smart monitoring of events like in $[4,5,13,16]$. Low-cost wireless sensors are deployed into a parking area which detect and monitor vacant parking slots. The events detected by sensor nodes are reported periodically to the management system. The database is accessed by an upper layer management system to perform various management functions, such as finding vacant parking lots, auto-toll, security management, and statistic reports. The types of wireless sensors suitable for parking systems and its performance are studied by researches [4, 12]. A comparison of data from different sensors to select the most appropriate method in detecting cars for WSN-based parking system is presented in [12]. An analysis of link characteristics in the car park scenario is studied in [4]. The experiments shows unexpected reliability patterns which have a strong influence on MAC and routing protocol design. In [11], a centralized method in assisting the car owners using a midrange wireless sensors and an opportunistic approach where a car shares the parking area information about the vacant slots are compared. Also, in [13], collaboration of cars is studied for efficient dissemination of information about parking spaces. The reports from wireless sensor nodes in [13] are passed from car-to-car in order to achieve scalable dissemination of information regarding parking spaces. Wireless sensor devices are used for automatic vehicle car parking [6] where wireless sensors and infrareds are used to maneuver the car in parking. The positioning and error correction procedures are calculated by the vehicles, and forwarded to the server. However, there is no means to identify the car parked in a specific slot. The identification of car presence detector cannot be associated with the car's identification using RFID tags where the system cannot tell exactly the identification of car that parked on a slot.

\subsection{Location techniques using radio signals}

Positioning techniques are important for monitoring the location of a mobile object. There are varieties of positioning techniques like global positioning system (GPS) [7] which accurately locates mobile or immobile objects. However, GPS is limited at outdoor measurements and cannot be used indoors. The time of arrival (TOA), angle of arrival (AOA), and received signal strength (RSS) can used at indoors. These are more appropriate at a short distance measure and less sophisticated compared to GPS. Both TOA and AOA are more complex and costly compared to RSS. RSS only needs to measure signal strength from the source and then calculates the position based on multilateration [10] which does not require complex design and additional hardware. Active RFID is used for location system that uses active signals [9]. Active badge location system [17] is one of the first indoor location system using active RFID. In [19], ultrasonic sensor is used for the location system. Studying the properties and determining the appropriate settings from these methods are also needed to be considered before implementing. In the proposed method, the location technique uses RSS patterns from CARFID and presence detector sensors. The calculation is presented in (1), where $P_{t x}$ is the transmission power, $\alpha$ is the path loss model, $P_{r c v d}$ is the signal strength is used to solve the $r$ or distance:

$P_{r c d}=c \frac{c P_{t x}}{r^{\alpha}}, \quad r=\alpha \sqrt{\frac{c P_{t x}}{P_{r c d}}}$.

The distance value from (1) can be used in the lateration method where the RSS coming from CARFID are gathered and at least three receiver nodes are needed. Pattern recognition of signal also provides a convenient way of implementing the localization in which it does not need sophisticated devices [15]. A study using nearest neighbor technique for RSS pattern recognition is proposed in [2]. Assume that RSS in any training location $\left(x_{i}, y_{i}\right)$ is a random variable $p$ with a density function $f\left[p /\left(x_{i}, y_{i}\right)\right]$ drawn from the samples of measurements performed at that location. Then, for a new measurement, $\operatorname{RSS}=P$ at an unknown location $(x, y)$, the probability of having that measurement taken in the specific training points $\left(x_{i}, y_{i}\right)$ is shown in (2). The actual estimation of an unknown location $\left(x_{i}, y_{i}\right)$ is the statistical average over all measured training locations in (3). Most of statistical methods are computational costly. Also, because of the unknown path loss model from RSS and obstructions, the calculation can be inaccurate. Approaches using nonlinear methods like neural-networks for the location technique based on classification of radio signal patterns can be effective and convenient:

$p_{i}=f\left[p /\left(x_{i}, y_{i}\right)\right]$,

$(x, y)=\sum_{i=1}^{N} p_{i}\left(x_{i}, y_{i}\right)$

Artificial neural network (ANN) is a computational system consisted of interconnected processing elements called neurons that process information as a response to external stimuli. A neuron contains a threshold value that regulates its action potential. ANN is used in many applications such as in the medical domain for clinical diagnosis image analysis and interpretation, and drug development. In radio signals, ANN is popularly used in cognitive communications [14]. MLP is a feedforward ANN model which maps a set of input data into a set of appropriate output using the multiple layers of neurons in a directed graph, with each layer fully connected to the next one. Commonly, MLP utilizes 


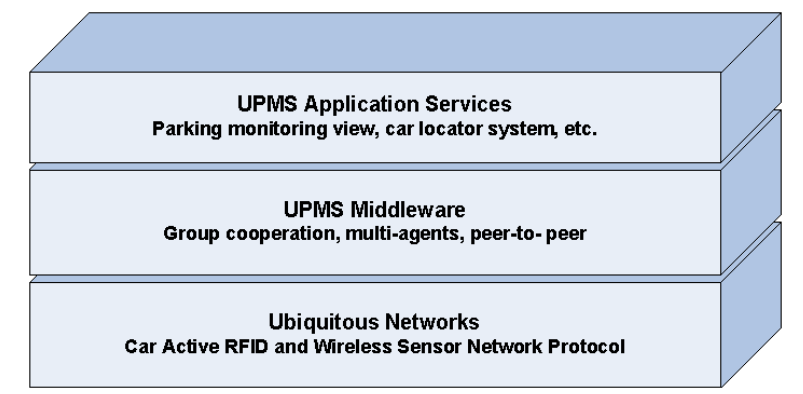

Figure 1: Framework for the ubiquitous parking management system (UPMS) consisting of three layers: application services, middleware, and ubiquitous network.

a supervised learning technique called backpropagation for training the network. In [1], the study uses a Modular MultiLayer Perceptron (MMLP) approach to effectively reduce the uncertainty in location estimation system where, in the simulation, that it obtained better result of location estimation than other approaches. ANN based method in location technique provides a cost-effective approach. In our proposal, the classification method of MLP is used to recognize the signals associated with the location of the presence detector.

\section{Architecture of the WSN-based parking management system}

The architecture of the parking management system using RFID and WSN is shown in Figure 1. The architecture is divided into three layers: ubiquitous network, middleware, and application services layers. The ubiquitous network represents the physical network of different sensors and computers communicating in the wireless environment. The components in the middleware layer are transparently executing for the efficient management of data gathered from the ubiquitous network layer. The interaction of clients and application services are also handled in the middleware layer. The application service layer is consisted of services for parking management system. This paper proposes a location technique using active RFID in the ubiquitous network layers of the parking management system. Unlike other location methods, the proposed design is not complex and cost-effective by only using wireless sensors to locate the car. Moreover, the proposed system uses multi-agent approach to promote intelligent dissemination of messages within the system.

Figure 2 is emphasizing a two-level parking area using the following devices: car presence sensor, RFID readers, parking display, wireless access controller, digital video recorder, and CARFID with motion sensor. Car owners will be provided with a CARFID before entering the parking area. Before a car enters the parking area, a display of available slot is projected in the screen of the parking display monitor. On entering the parking area, the RFID readers scan the identification of car. The drivers will be guided by the light indicators on top of parking slots which indicates vacant (green) or not (red). After choosing an empty slot, a car that has parked is detected by a car presence sensor. The car presence sensor sends a message to the wireless access controller to record the parking event and to store the data which includes the time of parking, car presence sensor ID and identification of the car. A car owner should turn-on the motion sensor after parking and leaving the car, and then turn-off before exiting the parking area. Also, a digital video recorder records the video of parking events inside the parking area which is also used in collision verification method. To identify a car, a camera is installed in the gate to get the image of the plate number.

In Figure 3, each presence sensor/detector transmits message to process in the server to log the parking event using the wireless network. On the other hand, smart phone and display devices use the Internet to request information. Agents in the UPMS middleware are shown at the bottom of Figure 3. The UPMS middleware uses multi-agent approach to provide an efficient information dissemination within the system. The multi-agent components are defined as follows:

(1) Parking Manager Agent-stores the information gathered from all agents and processes the request of information of an agent.

(2) Pattern Recognizer Agent-used in location technique for cars inside the parking area.

(3) Collision Analyzer Agent-detects and verifies the collision inside the parking area. The result from detection and verification processing is sent to the PMA to inform about the event to the affected car owners.

(4) Monitor Agent - displays the current available slots and other information for the car that entering the parking area.

(5) Event Notifier Agent-informs the owner of the important events inside the parking area.

\section{Location technique based on RSS pattern recognition}

A car is assigned with a CARFID and every time a car parked, the CARFID interacts with the wireless sensors in the parking area. Each wireless sensor which is used for presence detection of cars determines the RSS from CARFID and then sends the value to the sink node. In this proposal, RSSs are used for the recognition method from the trained structured of MLP. The proposed pattern recognizer agent gathers all RSSs and determines the slot location of car through classification method. Figure 4 shows the procedure of gathering RSS in each wireless sensor; (1) sending of data by CARFID; (2) each wireless sensor determines RSS from CARFID then send the values to the sink node; and (3) processing of RSSs using pattern recognizer agent based on MLP. 


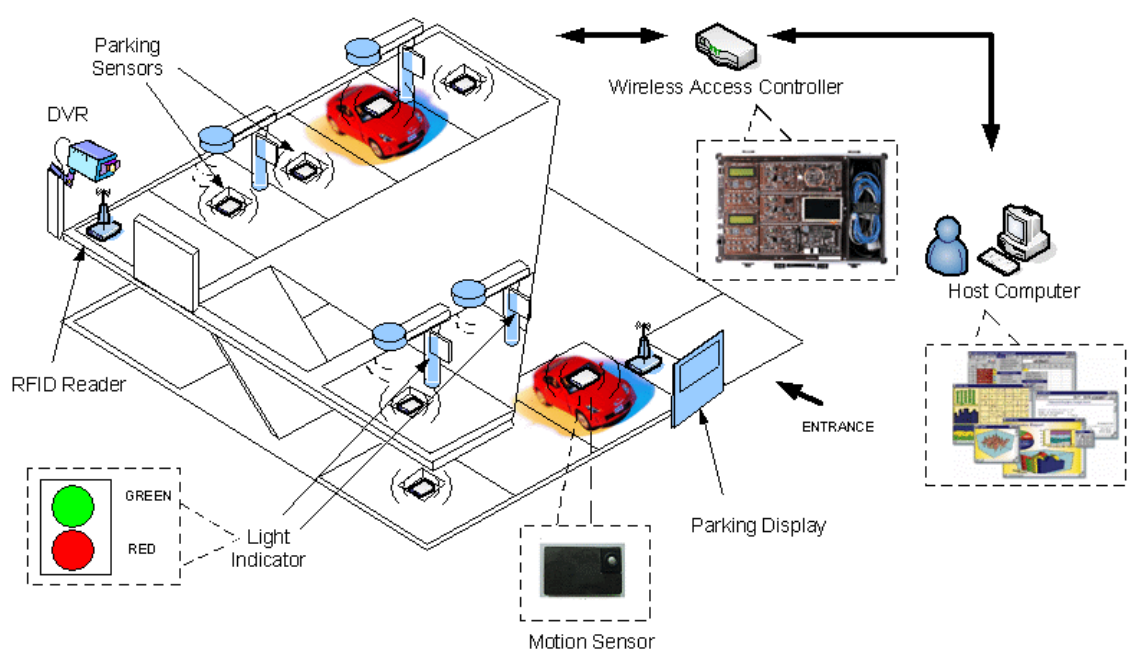

Figure 2: Ubiquitous network components in a two level parking establishment.

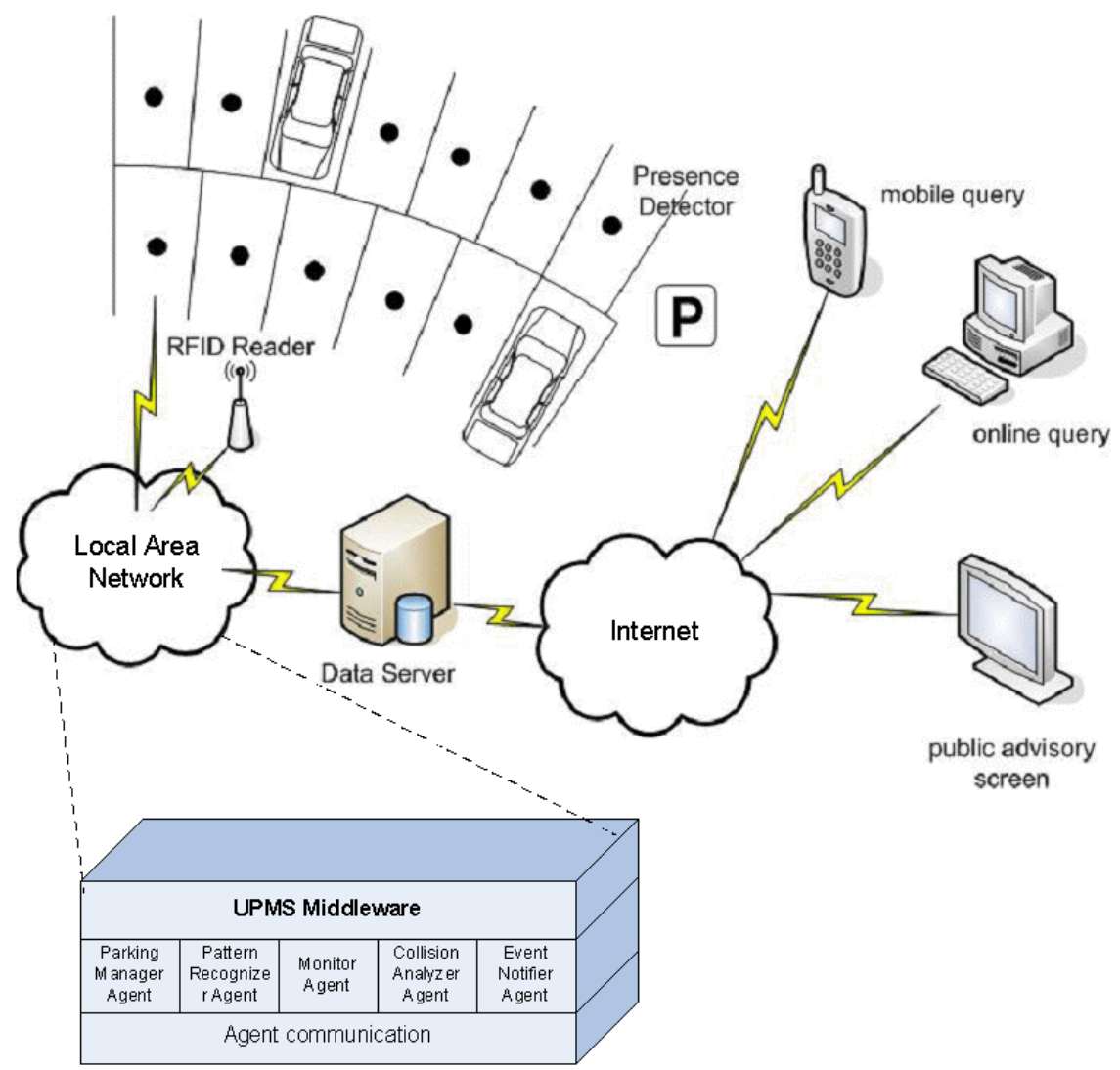

Figure 3: Interaction of the component devices in parking events based on the UPMS middleware.

\subsection{Car presence detector sensors}

A presence detector sensor is a wireless sensor used to detect car presence where light and temperature are used. The outputs from these sensors are processed in the adaptive inference engine (AIS). The AIS consists of membership function, inference rule and operators:
(1) Membership function - processes transformation of real values from sensors to categorical values to be processed in inference engine.

(2) Inference engine - contains a list of rules to determine the car presence.

(3) Operators - set of operators to process the rules in the inference engine. 


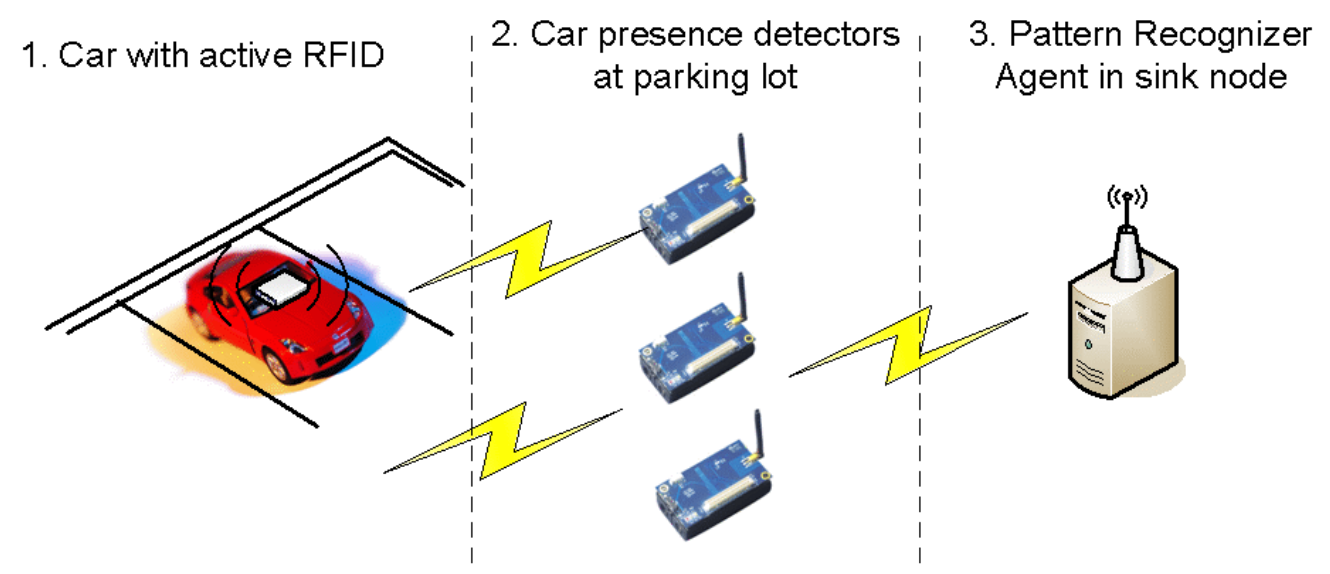

Figure 4: The procedure of gathering and processing the received signal strength (RSS).

Table 1: Rules used by the sensor module.

\begin{tabular}{cccc}
\hline Rule & Temperature $\left(S_{1}\right)$ & Luminance $\left(S_{2}\right)$ & Car presence $(C)$ \\
\hline 1 & High & Dark & Present \\
2 & Medium & Dark & Present \\
3 & High & Medium-bright & Present \\
4 & Medium & Medium-bright & Not present \\
5 & Low & Medium-bright & Not present \\
6 & Medium & Bright & Not present \\
7 & Low & Bright & Not present \\
\hline
\end{tabular}

The inputs of a single data sensor module are processed by AIS and then the output value is sent to the sink node. The AIS adapts the rules from the adaptive module in the sink node. Rules are configured by expert based on the environment of the parking area. The sensor inputs $(S)$ from presence detector sensor are processed in the AIS. The $S_{i}$ is a categorized value which was processed in the membership function where $i$ is the index of the sensor input. The values are gathered at the same time, $t_{n}=\left\{S_{1}, S_{2}, \ldots, S_{i}\right\}$, and processed in the AIS to determine the presence of car represented by $C$. Two input parameters are defined for the rules of the AIS, temperature $\left(S_{1}\right)$ and luminance $\left(S_{2}\right)$. The presence of car is detected from rules 1 to 3 while car is not present from rules 4 to 7 shown in Table 1 .

Extreme conditions in the parking area could affect the values of sensor readings. An adaptive module is implemented to adjust the values in the AIS. The sink node is provided with the adaptive module which is configured based on the state of environment in the parking area. Threshold values are used to trigger the recon in the sink node. The new configurations from AIS are used to process the inputs from the sensors to provide correct car presence detection. Rules are defined by expert based on the conditions of the car parking where $R=\left\{R_{1}, R_{2}, \ldots, R_{k}\right\}$ :

$R_{k}=\mathrm{IF} S_{1}$ is $A_{1}$ AND,$\ldots$, AND $S_{k}$ is $A_{k}$ then $\sigma_{k}$.
The outcome of the rule in (4) is a change value $\left(\sigma_{l}\right)$ to the parameters of the AIS and will be transmitted to all sensors in the parking area after the changes in the parking environment are determined by the adaptive module. These values are sent in wireless transmission.

\subsection{RSS pattern recognition}

After a presence detector sensor detects a car on a slot, it triggers the sending of message to CARFID. The CARFID receives the information from the presence detector sensor and it starts sending information to all sensors. The information from the CARFID will be received by each sensor to get the RSS values. The location method of PRA starts gathering the RSS and classifies it using the MLP structure. Equation (5) is the function of choosing the parking slot associated to the RSS patterns where $p_{n}$ is a parking slot and $x$ is an input vector of RSS. The result with the maximum value from the aggregation is the chosen parking slot associated to the radio signals. Moreover, the details of processing function together with the weight value $(w)$ from the MLP structure is presented in (6). The output value from processing the product of $x$ and $w$ is returned to the PRA to decide the slot location:

$$
\begin{aligned}
\text { parking_slot } & =\max \left\{\sum_{n=1}^{N} p_{n}(x)\right\}, \\
p_{n}(x) & =\sum_{j=1}^{J} x_{j} w_{j} .
\end{aligned}
$$

\subsection{Training MLP with RSS pattern}

RSS patterns are trained in the MLP of the proposed pattern recognizer agent. MLP is consisted of three layers of processing nodes; an input layer which accepts the input variables used in the classification procedure, one hidden layers, and an output layer with one node per class. Equation (7) shows the over all calculation of MLP. RSS patterns are 
presented as input $x$ and weight value of the connection as $w$. The $z_{k}$ refers to the output value from calculating the activation function from input to hidden and hidden to output nodes:

$z_{k}=f\left(\sum_{j=1}^{n H} w_{k j} f\left(\sum_{j=1}^{d} w_{j i} x_{i}+w_{j 0}\right)+w_{k 0}\right)$.

The back-propagation algorithm is a gradient descent optimization procedure which minimizes the mean square error between the network's output and the desired output for all input patterns $P$. The outputs are compared to the target values and the differences are determined. The error is minimized by the function when every network outputs match the desired outputs. The least mean square (LMS) for two layer nets is shown in (8), where $t$ and $z$ are the target and the network output vectors of length $c$ and $w$ represents all the weights in the network:

$J(w)=\frac{1}{2} \sum_{k=1}^{c}\left(t_{k}-z_{k}\right) 2=\frac{1}{2}\|t-z\|$.

\subsection{Managing misclassified patterns}

After training, the structure of MLP is used to recognize the pattern of signals to determine the car identification and verifies the slot location of car. First, the RSS patterns from a new arrived car are processed in MLP and the proposed system produces the output. The output is compared to the presence detector sensor that detected the car. If the output and the sensor ID is the same then it reports to the monitoring program of the location of car. Else, if it is not the same, the input patterns are again processed in the training.

\section{Results and analysis}

Our design of the parking simulator platform used wireless sensor motes which are $2.4 \mathrm{GHz}$ and IEEE 802.15.4 complaints of its RF module. The FIS is coded in each wireless sensor mote which used nesC programming language. The adaptive rule base module from the sink node was programmed in Java. In simulating parking events, the active RFID was put on top of the car model shown in Figure 5. Every time a car parked on a parking slot, the wireless sensor under that slot detected the car and sent message to the active RFID on top of the car model to initialize the location technique as discussed in Section 4.2.

The CARFID prototype is shown in Figure 6. The area of the CARFID is $8.5 \mathrm{~cm}$ by $5.5 \mathrm{~cm}$ which is equal to the size of an ATM card and the thickness is about $1 \mathrm{~cm}$. We used the same radio frequency of the wireless sensor motes to communicate with CARFID. In Figure 6(a), CARFID has a command button to represent other functions like automated opening doors at the entrance of parking lot. Figure 6(b) shows the hardware inside of CARFID. CARFID is

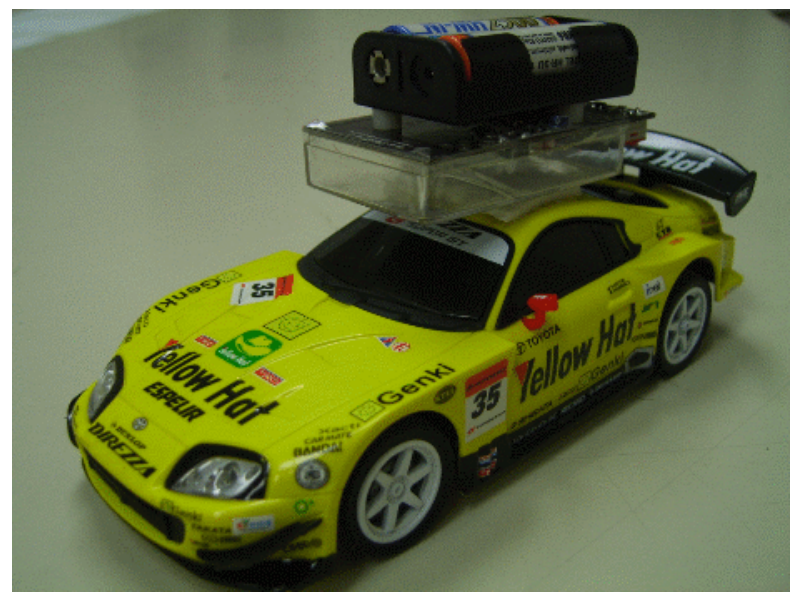

Figure 5: The active RFID is placed on the top of the car model.

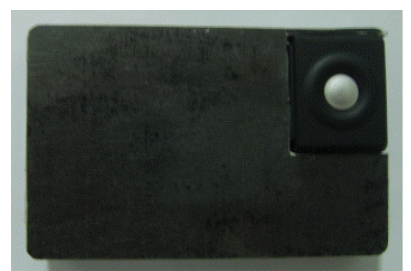

(a)

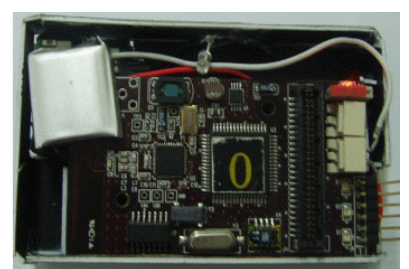

(b)
Figure 6: The front side (a) and back side without cover (b) of the CARFID.

provided with a small sized battery and like the presence detector sensor, the commands and interaction are also programmed in nesC. The program of CARFID, to interact with the wireless sensors was coded and designed in nesC as well. PRA which was used to receive and process the RSS was programmed in Java. The IDs of each presence detector was associated with the slot location and CARFID was used for the car identification.

Figure 7 presents the configuration for adaptive rule base module and training of RSS patterns in MLP. A GUI program was developed to do these tasks and other services. On the parent window, the child window for training the MLP is shown at the left of Figure 7. The training of RSS was performed in three steps. First, gathering of RSS data patterns by performing parking events. This procedure includes parking the car in each parking slot and records the signals that are associated to the slot that is currently occupied. After RSS patterns were gathered, all patterns were used to train MLP. The last step is to test the trained MLP in classifying current RSS. The inferencing system and rules from the adaptive rule base module are modified in another child window at the right of the parent window in Figure 7.

The simulation of parking events in the parking simulator platform is presented in Figures 8 and 9. The 


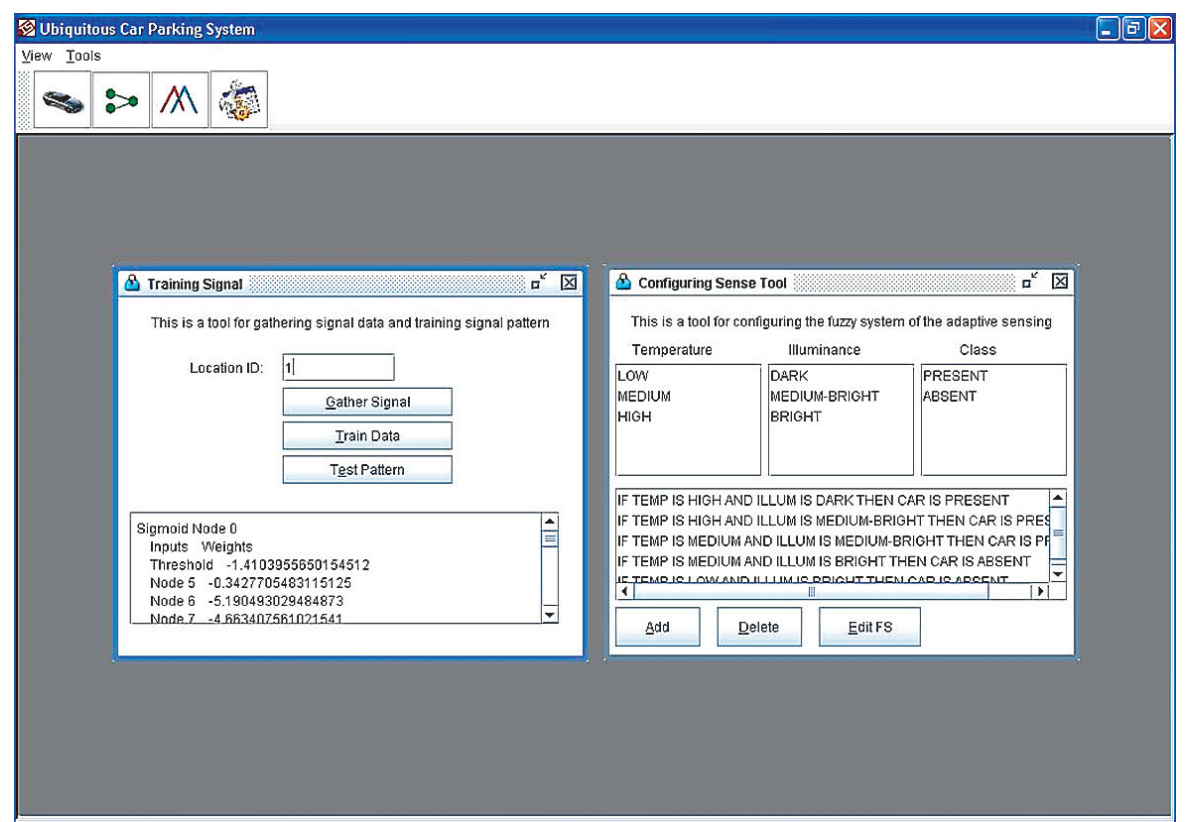

Figure 7: The configuration of adaptive rule base module and MLP training are performed in the GUI program of ubiquitous parking management system.

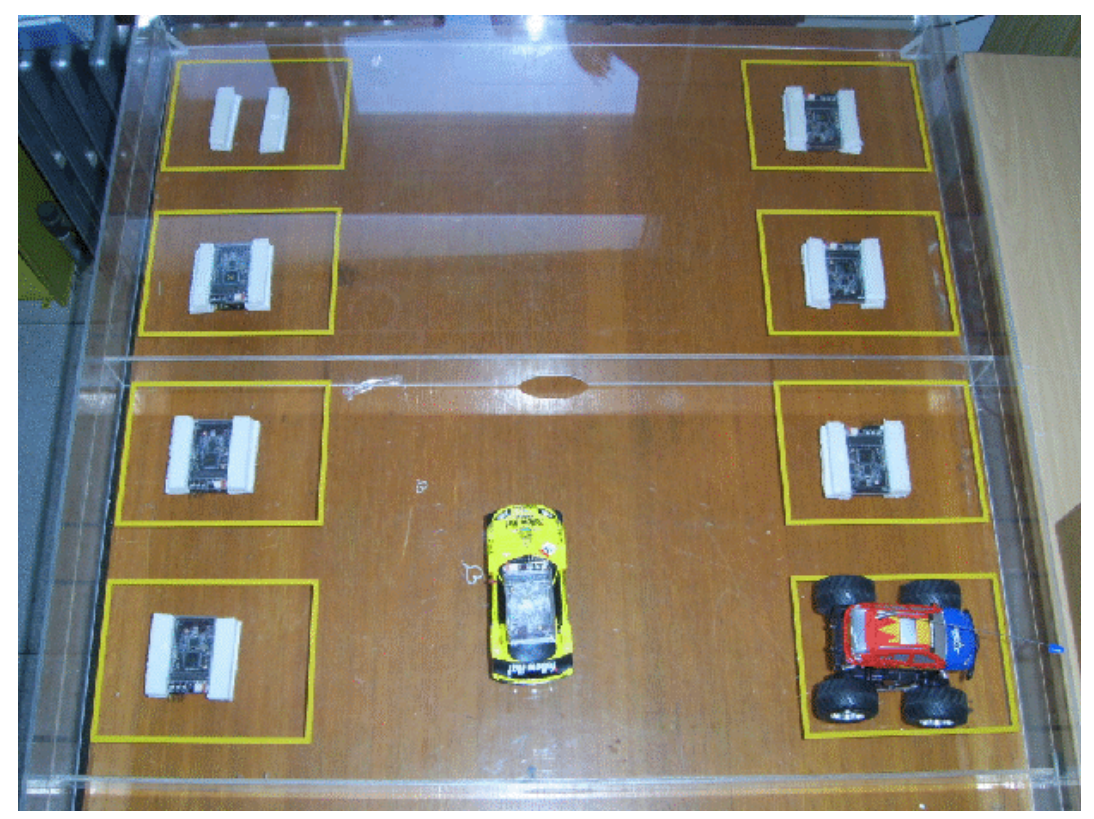

Figure 8: The car parking simulator platform consists of 8 wireless sensor motes placed under the platform.

area of parking simulator platform is $120 \mathrm{~cm} \times 90 \mathrm{~cm}$ which was placed inside of an office type room. Each slot area is $20 \mathrm{~cm} \times 15 \mathrm{~cm}$ with a boundary of $3.5 \mathrm{~cm}$ between each slot and drive way of $36 \mathrm{~cm}$. The platform is consisted of 8 slots and embedded with wireless sensors under each slot. Figure 8 illustrates a car parking in a single slot and at the same time the GUI program displays that the car is parked in slot no. 4 shown in Figure 9. The output display was updated every time and the values were stored in the database. By using the CARFID to identify each car, the problem of simultaneous parking of cars was solved. Each sensor determined the RSS from CARFID and sent the values to the sink node. The pattern recognizer agent gathered RSS data and associated the slot address when a car presence was detected. After knowing the identification of car, this information was stored in the system database. 




Figure 9: The car parking program simulator displays the output from the platform.

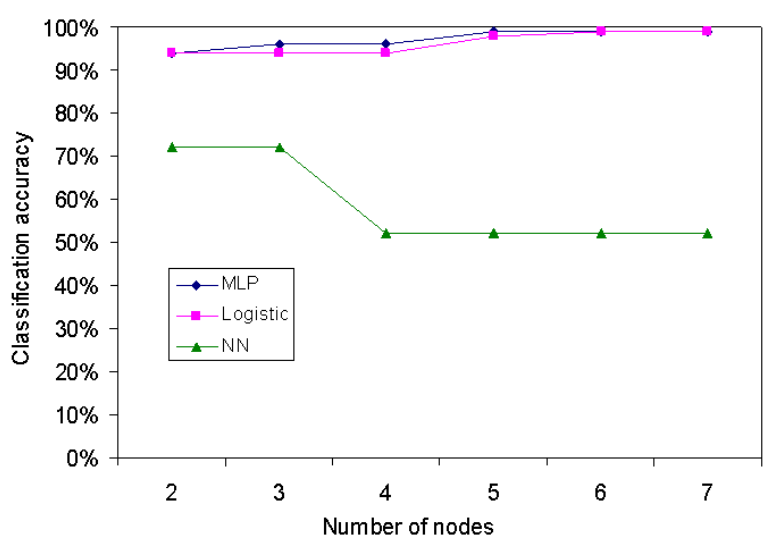

Figure 10: Processing time to build the model of the MLP, logistic and NN algorithms.

\subsection{Performance evaluation}

Several path loss models are considered in RSS location techniques [15] and our simulation environment considered the office space type. We used a maximum of 7 nodes for simulating the proposed location technique and a car model which is a scale of 28 times smaller than the original size. In the simulation, each RSS readings from the nodes were recorded and stored in the database whenever a car was on the slot area. We selected 100 patterns containing five different locations classes with RSS signals where each location class contains 20 patterns. Most studies measured the distance error, however, this study focused more on the pattern recognition where the accuracy of classifying patterns

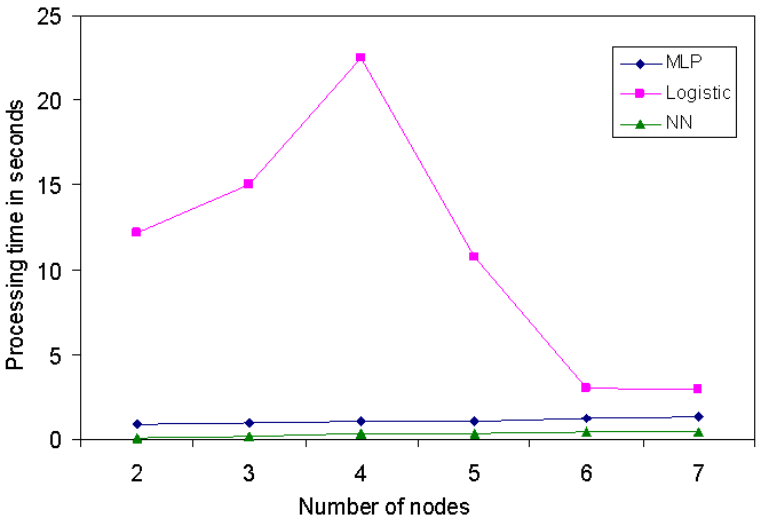

Figure 11: Accuracy of the MLP, logistic and NN algorithms in classifying the patterns from RSS.

from the current signals was observed. The cross-validation method is a popular classification test which uses the same training data in determining the number of misclassified patterns and is used for our evaluation. The nearest neighbor (NN) based on averaging the group of patterns and logistic regression which is a statistical model for probability modeling of patterns were compared.

Figure 10 shows that MLP and logistics are more accurate in classifying the patterns compared to NN. Also, by increasing the number of nodes, MLP and logistic classification accuracy increases while the NN decreases. However, Figure 11 shows that MLP is efficient in processing time compared to logistic. Building the statistical model of 
logistic is computational costly and by adding another node between 2 to 6 increases the calculation time. MLP showed more efficient in processing time than logistic regression. Even though MLP was not faster than NN, it had a big difference in accuracy shown in Figure 10. In overall, the pattern recognizer agent has an average of $97 \%$ of accuracy which was more accurate in classifying RSS patterns and time efficient compared to nearest neighbor and logistic regression, respectively.

\section{Conclusion}

The use of active RFID provides flexibility to application in wireless environment. Moreover, the main challenges here are to considered efficient and inexpensive location technique to implement for the system. This paper proposed a location technique based on pattern recognition of radio signals for the ubiquitous parking management system. To accurately locate a slot location of car using received signal strength (RSS) from each parking sensor, the pattern recognizer agent (PRA), based on multilayer perceptron, was proposed. The proposed method was simulated in the parking system simulator. The result of performance evaluation showed that using MLP to PRA acquires accurate classification of current signals to identify the car that parked in a slot.

Acknowledgment This research is supported by Industry and University Research Consortium of Small and Medium Business Administration (SMBA) of 2011.

\section{References}

[1] U. Ahmad, A. Gavrilov, U. Nasir, M. Iqbal, S. J. Cho, and S. Lee, In-building localization using neural networks, in Proc. IEEE International Conference on Engineering of Intelligent Systems, Islamabad, Pakistan, 2006, 1-6.

[2] P. Bahl and V. N. Padmanabhan, RADAR: an in-building RFbased user location and tracking system, in Proc. 19th Annual Joint Conference of the IEEE Computer and Communications Societies (INFOCOM' 00), vol. 2, Tel Aviv, Israel, 2006, 775784.

[3] E. Barata, L. Cruz, and J.-P. Ferreira, Parking at the UC campus: Problems and solutions, Cities, 28 (2011), 406-413.

[4] J. P. Benson, T. O’Donovan, P. O'Sullivan, U. Roedig, C. Sreenan, J. Barton, et al., Car-park management using wireless sensor networks, in Proc. 31st Annual IEEE Conference on Local Computer Networks, Tampa, FL, 2006, 588-595.

[5] L. F. Cervantes, Y.-S. Lee, H. Yang, and J. Lee, A hybrid middleware for RFID-based parking management system using group communication in overlay networks, in Proc. International Conference on Intelligent Pervasive Computing (IPC '07), Jeju Island, Korea, 2007, 521-526.

[6] L. Chou, D. C. C. Sheu, and H. W. Chen, Design and prototype implementation of a novel automatic vehicle parking system, International Journal of Smart Home, 1 (2007), 11-16.

[7] B. Hoffmann-Wellenhof, H. Lichtenegger, and J. Collins, Global Positioning System: Theory and Practice, Springer-Verlag, New York, 5th ed., 2001.

[8] A. Ibeas, R. Cordera, L. dell'Olio, and J. L. Moura, Modelling demand in restricted parking zones, Transportation Research Part A: Policy and Practice, 45 (2011), 485-498.
[9] G.-Y. Jin, X.-Y. Lu, and M.-S. Park, An indoor localization mechanism using active RFID tag, in Proc. IEEE International Conference on Sensor Networks, Ubiquitous, and Trustworthy Computing, vol. 1, Taichung, Taiwan, 2006, 1-4.

[10] H. Karl and A. Willig, Protocols and Architectures for Wireless Sensor Networks, John Wiley \& Sons, New York, 2005.

[11] E. Kokolaki, M. Karaliopoulos, and I. Stavrakakis, Opportunistically assisted parking service discovery: Now it helps, now it does not, Pervasive and Mobile Computing, 8 (2012), 210-227.

[12] R. Kumar, N. K. Chilamkurti, and B. Soh, A comparative study of different sensors for smart car park management, in Proc. International Conference on Intelligent Pervasive Computing (IPC '07), Jeju Island, Korea, 2007, 499-502.

[13] S. Miura, Y. Zhan, and T. Kuroda, Evaluation of parking search using sensor network, in Proc. 1st International Symposium on Wireless Pervasive Computing, Phuket, Thailand, 2006, 1-6.

[14] O. Orcay and B. Ustundag, Pattern recognition in cognitive communication, in Proc. 23rd International Symposium on Computer and Information Sciences (ISCIS '08), Istanbul, Turkey, 2008, 1-6.

[15] K. Pahlavan and A. H. Levesque, Wireless Information Networks, John Wiley \& Sons, New York, 2nd ed., 2005.

[16] V. W. S. Tang, Y. Zheng, and J. Cao, An intelligent car park management system based on wireless sensor networks, in Proc. 1st International Symposium on Pervasive Computing and Applications, Urumqi, Xinjiang, China, 2006, 65-70.

[17] D. Teodorović and P. Lučić, Intelligent parking systems, Eur. J. Oper. Res., 175 (2006), 1666-1681.

[18] R. Want, A. Hopper, V. Falcão, and J. Gibbons, The active badge location system, ACM Trans. Inf. Syst., 10 (1992), 91-102.

[19] A. Ward, A. Jones, and A. Hopper, A new location technique for the active office, IEEE Personal Communications, 4 (1997), 4247. 